\title{
Finding schizophrenia genes
}

\author{
George Kirov, Michael C. O’Donovan, and Michael J. Owen
}

Department of Psychological Medicine, Wales College of Medicine, Cardiff University, Cardiff, United Kingdom.

\begin{abstract}
Genetic epidemiological studies suggest that individual variation in susceptibility to schizophrenia is largely genetic, reflecting alleles of moderate to small effect in multiple genes. Molecular genetic studies have identified a number of potential regions of linkage and 2 associated chromosomal abnormalities, and accumulating evidence favors several positional candidate genes. These findings are grounds for optimism that insight into genetic factors associated with schizophrenia will help further our understanding of this disease and contribute to the development of new ways to treat it.
\end{abstract}

Schizophrenia is a disorder characterized by delusions, hallucinations, reduced interest and drive, altered emotional reactivity, and disorganized behavior. Often, cognitive and behavioral signs are present from early childhood, but the characteristic features generally start in the late teens and early twenties. Although outcomes are variable, even with treatment, the typical course is one of relapses followed by only partial remission as well as a marked reduction in social and occupational function such that sufferers are often the most vulnerable, isolated, and disadvantaged individuals in society. In a recent metaanalysis the median incidence rate was 15.2 per 100,000 , with the $10 \%$ to $90 \%$ quantiles between 7.7 and 43.0 per 100,000 (1).

\section{Genetic epidemiology}

The results of numerous family, twin, and adoption studies show conclusively that risk of schizophrenia is increased among the relatives of affected individuals and that it is the result largely of genes rather than shared environment (2-4). In the children and siblings of individuals with schizophrenia, the increase in risk is approximately 10-fold; it is somewhat less than this in parents (Figure 1).

The latter finding is probably explained by a reduction in the reproductive opportunities, drive, and possibly the fertility of affected individuals. Five recent systematically ascertained studies report monozygotic concordance estimated at 41-65\% compared with dizygotic concordance of $0-28 \%$ and an estimate of broad heritability of $85 \%(5)$. Among complex genetic disorders, schizophrenia has one of the highest heritabilities. To place this in perspective, the heritability of schizophrenia is similar to that of type I diabetes (72\%-88\%; refs. 6,7$)$, but greater than that of breast cancer (30\%; ref. 8), coronary heart disease in males (57\%; ref. 9), and type II diabetes (26\%; ref. 10$)$.

While the twin and adoption literature leave little doubt that genes are important, they also point to the importance of environmental factors, since the concordance for schizophrenia in monozygotic twins is typically around $50 \%$ and heritability estimates are less than $100 \%$. Moreover, we should also note that

Nonstandard abbreviations used: $A R V C F$, armadillo repeat deleted in velocardiofacial syndrome gene; $C O M T$, gene encoding catecholamine O-methyl transferase; $D A O$, gene encoding $\mathrm{D}$-amino acid oxidase; $D A O A$, gene encoding $\mathrm{D}$-amino acid oxidase activator; DTNBP1, dystrobrevin-binding protein 1; ErbB, v-erb-b2 erythroblastic leukemia viral oncogene homologue; GRM3, gene encoding metabotropic glutamate receptor type 3; LD, linkage disequilibrium; NMDA, N-methly-D-aspartate; NRG1, gene encoding neuregulin 1; $P R O D H$, gene encoding proline dehydrogenase; RGS4, gene encoding regulator of $G$ protein signaling $4 ; \lambda_{S}$, sibling relative risk; SNP, single nucleotide polymorphism; TRAR4, gene encoding trace amine receptor $4 ; Z D H H C 8$, gene encoding zinc finger- and DHHC domain-containing protein 8.

Conflict of interest: The authors have declared that no conflict of interest exists.

Citation for this article: J. Clin. Invest. 115:1440-1448 (2005).

doi:10.1172/JCI24759. risks resulting from some gene-environment interactions tend to be attributed to genes in most genetic epidemiological studies.

It is clear from genetic epidemiology that the mode of transmission is complex $(11,12)$. However, the number of susceptibility loci, the disease risk conferred by each locus, the extent of genetic heterogeneity, and the degree of interaction among loci all remain unknown. Risch (13) has calculated that the data are incompatible with the existence of a single locus conferring a sibling relative risk $\left(\lambda_{S}\right)$ of more than 3 and, unless extreme epistasis exists, models with 2 or 3 loci of $\lambda_{S}$ less than or equal to 2 are more plausible. It should be emphasized that these calculations are based upon the assumption of homogeneity and refer to population-wide $\lambda_{\mathrm{S}}$. It is quite possible that alleles of larger effect are operating in some groups of patients, for example, families with a high density of illness. However, high density families are expected to occur by chance even under polygenic inheritance, and their existence does not prove the existence of disease alleles of large effect (12).

\section{The phenotype of schizophrenia}

Different combinations of symptoms exist in different individuals, and there is considerable heterogeneity of disease course and outcome. However, it is not known whether schizophrenia is a single disorder with variable clinical manifestations or a group of syndromes, each with a unique or overlapping pathophysiology. In spite of this, structured and semistructured interviews together with explicit operational diagnostic criteria permit high diagnostic reliability. Given that clinically diagnosed schizophrenia has a high heritability, it should, in principle, be possible to subject it to molecular genetic analysis. However, schizophrenia, as defined by current diagnostic criteria, may well include a number of heterogeneous disease processes, in which case attempts at identifying genes and other etiological factors would be greatly facilitated if it were possible to distinguish reliably among them (14).

The situation is further complicated by the fact that we are unable to define the limits of the clinical phenotype to which genetic liability can lead. It clearly extends beyond the core diagnosis of schizophrenia to encompass a spectrum of disorders, including schizoaffective disorder and schizotypal personality disorder $(15,16)$. However, the limits of this spectrum and its relationship to other psychoses, affective and nonaffective, and nonpsychotic affective disorders remain uncertain $(17,18)$.

One of the earliest benefits of the identification of susceptibility genes is that the validity of current nosological categories can be further explored. Knowing the genes involved should illuminate heterogeneity within the current concept of schizophrenia and help us to understand its relationship to other diagnostic groups. 


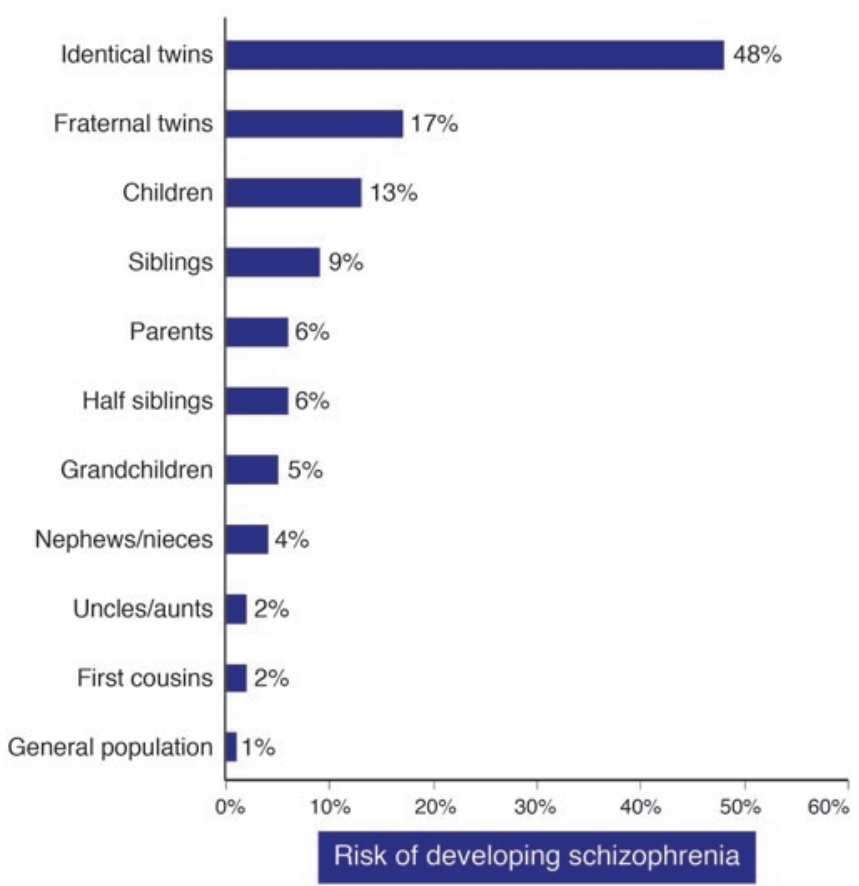

Figure 1

Risk of developing schizophrenia in relatives of schizophrenic probands. The data are based on the review of studies compiled by Gottesman (3).

\section{Epidemiology, pathophysiology, and neurobiology}

Epidemiological studies have identified a number of environmental factors associated with schizophrenia: pregnancy and delivery complications, delayed development, winter and spring birth, low IQ, urban birth and domicile, immigration, and the use of illegal drugs (especially cannabis; ref. 19). However, the effect sizes are small, and in most cases the direction of causation is uncertain.

For many years, ideas about the pathophysiology of schizophrenia were based on pharmacological studies. The classic "dopamine hypothesis," in which schizophrenia was attributed to a hyperdopaminergic state, derived from the realization that the potency of the first generation of antipsychotic drugs was proportional to their ability to block dopamine receptors (20). Modern refinements suggest a more complex picture, with increased dopaminergic transmission in the basal ganglia associated with acute psychosis (21) and a prefrontal cortical dopamine deficit responsible for chronic cognitive impairments (22). Psychopharmacological studies have also implicated altered serotonergic, and, more compellingly, glutamatergic transmission $(23,24)$. However, as these systems interact, the hypotheses are not mutually exclusive, and at least some of the dopaminergic changes may be secondary to altered cortical glutamatergic transmission (25).

The fundamental causes, whatever they are, appear to act during neurodevelopment rather than simply at the onset of the disorder $(26,27)$. This is supported by many of the epidemiological findings summarized above, by observations that children who subsequently develop schizophrenia have higher rates of neuropsychological and motor deficits, and by neuroimaging studies showing ventricular enlargement and reductions in cortical volume at illness onset (28) and in those at high risk of the disorder by virtue of increased genetic loading (29). The reduction in size of some brain structures shown by neuroimaging could in princi- ple be due to neurodevelopmental or neurodegenerative processes or a combination of the 2 . The existence of a neurodevelopmental mechanism is supported by the failure of neuropathological studies to detect markers of neurodegeneration (30). Neuropathological studies have also failed to establish a clear diagnostic neuropathology, and findings have often been conflicting. However, fairly consistent evidence has emerged for widespread nonuniform reductions in the neuropil and neuronal size, with the temporal lobe, prefrontal cortex, and dorsal thalamus particularly affected (30). These changes, together with reductions seen in synaptic and dendritic markers and abnormalities in white matter (31), suggest deficits in synaptic structure, function, and connectivity between neurons (30).

Epidemiological, pharmacological, and neurobiological studies have certainly brought about progress in our general understanding of schizophrenia. However, it is impossible confidently to implicate specific pathophysiological processes from the rather vague concepts of altered neurodevelopment, synaptic dysfunction, or neuronal connectivity. The more specific neurotransmitter-based hypotheses are very likely to be relevant to some of the overt clinical manifestations of schizophrenia, but with few exceptions, molecular genetic studies predicated on these hypotheses have met with disappointing results. This, together with the evidence for high heritability, has encouraged a number of groups to apply positional genetic approaches as these do not depend upon knowledge of disease pathophysiology.

Three main approaches have been used to seek genes for schizophrenia: positional genetics based on genome-wide linkage analysis, identification of chromosomal abnormalities associated with schizophrenia, and studies of functional candidate genes. In the following sections, we review the main findings that have emerged from each.

\section{Molecular genetic studies}

Linkage analysis. In contrast with several other complex disorders, such as breast cancer, Alzheimer disease, Parkinson disease, and epilepsy, no forms of schizophrenia following Mendelian inheritance patterns have yet been discovered. Indeed the results of linkage studies in schizophrenia have been disappointing, with positive studies often falling short of stringent genome-wide levels of significance and abundant failures to replicate. This is probably attributable to a combination of small genetic effects and inadequate sample sizes (32). However, as more than 20 genome-wide studies have been reported and sample sizes increased, some consistent patterns have emerged. Linkages that reached genome-wide significance on their own according to the criteria set forth by Lander and Kruglyak (33) and those that have received strong support from more than one sample are illustrated in Figure 2 (34-54).

Recently, 2 metaanalyses of schizophrenia linkage have been reported. Each used different methods and obtained overlapping but somewhat different results. The study of Badner and Gershon (55) supported the existence of susceptibility genes on chromosomes 8p, 13q, and 22q, while that of Lewis et al. (56) most strongly favored $2 \mathrm{q}$ but also found that the number of loci meeting the aggregate criteria for significance was much greater than the number of loci expected by chance $(P<0.001)$. Evidence was also obtained for susceptibility gene regions on chromosomes $5 \mathrm{q}$, $3 \mathrm{p}, 11 \mathrm{q}, 6 \mathrm{p}, 1 \mathrm{q}, 22 \mathrm{q}, 8 \mathrm{p}, 20 \mathrm{q}$, and $14 \mathrm{p}$. The $8 \mathrm{p}$ and $22 \mathrm{q}$ regions were supported by both metaanalyses, but 8 other regions were supported by only one. Furthermore the region most strongly sup- 


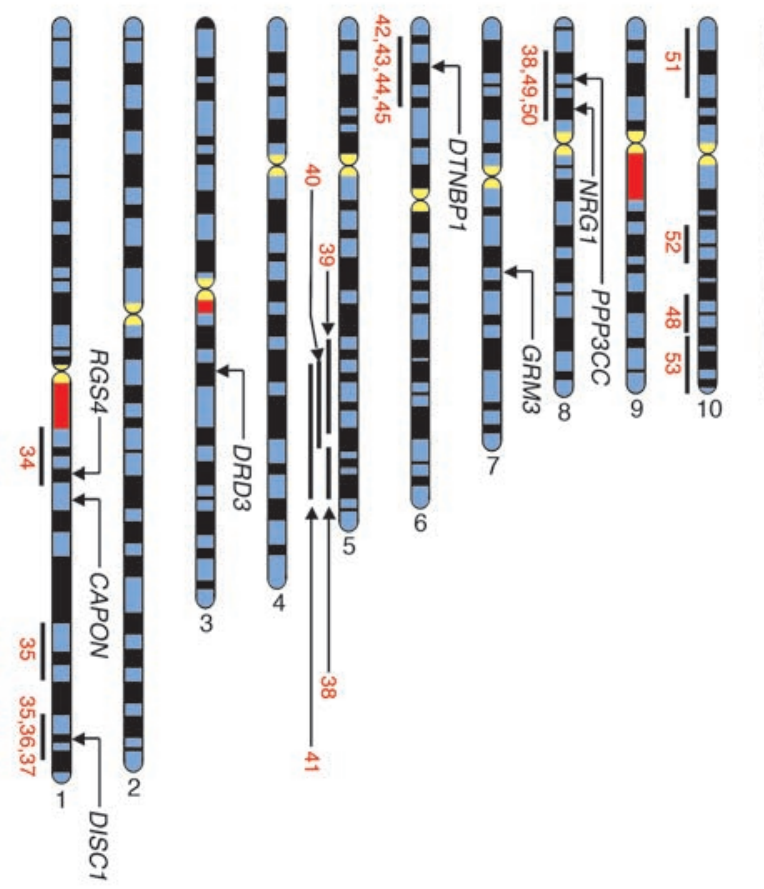

ported by the Lewis et al. (56) analysis, that on chromosome 2q, is not one that had received strong support previously; now it is clearly worthy of further investigation.

The linkage data therefore support the predictions made by Risch (13) on the basis of genetic epidemiological findings: it is highly unlikely that there exists a locus of effect size $\lambda_{\mathrm{S}}$ greater than 3 . However, there is evidence that implicates a number of regions, which is consistent with the existence of susceptibility alleles of moderate effect $\left(\lambda_{\mathrm{S}} 1.2-3\right)$ and possibly uncommon loci of larger effect that can be identified in specific samples of large multiply affected families. Of course, the proof that a positive linkage is correct comes when the disease gene, or genes, is identified, and a number of the linked regions are currently being subjected to detailed analysis with, as we shall see, encouraging results.

Positional candidate genetics. Previously, the economics and practicalities of hunting a susceptibility gene within a linked chromosomal region dictated that one should have virtually definitive evidence that the linkage was a true positive before beginning. With recent improvements in our knowledge of genome anatomy and in genome analysis technology, the task of positional cloning has been transformed and now favors bolder endeavors. These considerations, together with the convergence of some positive linkage findings, have led to a number of detailed mapping studies of linked regions which have in turn implicated specific genes. However, the quality of the data has been variable, and a number of putative susceptibility genes have yet to be clearly replicated. Here we focus on the genes for which, at the time of this writing, there are published follow-up studies or for which we are aware of data that allow judgment about whether the gene is likely to be a true positive. In making these judgments, we have primarily been influenced by the strength of the genetic evidence for association to the clinical phenotype. We have given no weight to claims that a single nucleotide polymorphism (SNP) is functional or that the gene has plausibility by virtue of patterns of expression or participation in relevant
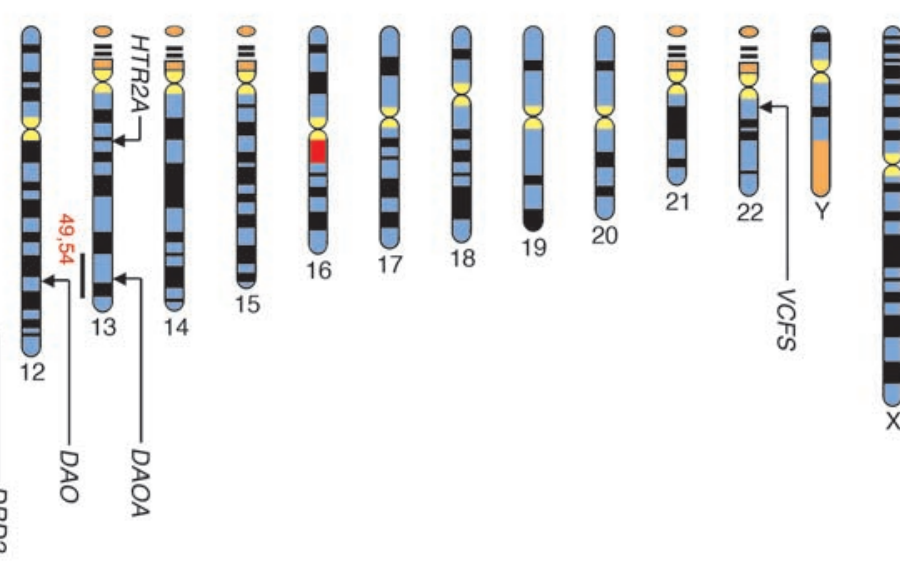

\begin{abstract}
Figure 2
Locations of linkage findings and genes discussed in this paper. Linkages that reached genome-wide significance on their own according to the criteria set forth by Lander and Kruglyak (33) or those that have received strong support from more than one sample are shown. The numbers in black are the numbers of the chromosomes. The numbers in red refer to the references.
\end{abstract}

pathophysiological pathways. These are key properties of true susceptibility variants, but the number of genes that meet the above criteria is so vast that such evidence cannot be used significantly to increase the prior probability that a gene is implicated in schizophrenia and to prop up weak genetic findings. We have also given little weight to associations with endophenotypes, be they based on electrophysiology, pharmacology, psychology, or neuroimaging or performed in humans or modeled in animals. Such studies are likely to be essential for understanding how variation in a proven susceptibility gene leads to the clinical phenotype. However, the use of endophenotypes for the identification of disease genes requires that the measures in question are trait, rather than state, variables as well as compelling evidence from genetic epidemiology that they reflect genetic vulnerability to that disease rather than environmental factors. Promising data are accumulating for some potential endophenotypes for schizophrenia (57), but, in most cases, uncertainties remain.

Dystrobrevin-binding protein 1. Evidence that the gene encoding dystrobrevin-binding protein 1 (DTNBP1) or dysbindin has a role in schizophrenia was first reported by Straub et al. (58), who undertook association mapping across the linkage region on chromosome $6 \mathrm{p} 22.3$. Significant associations were found between schizophrenia and several SNPs and multimarker haplotypes spanning DTNBP1. A second study based on 203 families, most of which came from Germany (59), provided further evidence for association between DTNBP1 and schizophrenia as did 2 large studies from our own group, which included approximately 900 schizophrenic patients from the United Kingdom and Ireland (60) and 488 parent-proband trios from Bulgaria (61). Although there have also been some studies in which no association was found $(62,63)$, significant associations in a total of 10 samples have now been published $(64,65)$. Moreover, significant association was found in the initially negative sample of Morris and colleagues (63), when 1 additional marker that defined the risk haplotype in our own UK sample was genotyped (60). 
Thus, the evidence in favor of DTNBP1 as a susceptibility gene for schizophrenia is strong. However, there are inconsistencies between the specific risk alleles and haplotypes among studies, which suggests the presence of multiple susceptibility and protective alleles (60) or the possibility that a single susceptibility allele is carried on a remarkable diversity of haplotypes even in closely related populations.

As yet, no causative variant has been identified, and the absence of associated nonsynonymous alleles after systematic mutation detection of the exons (60) suggests that disease susceptibility depends upon variation affecting mRNA expression or processing. The latter possibility is indirectly supported by evidence for as yet unknown cis-acting polymorphisms affecting $D T N B P 1$ expression in the human brain (66) and more directly supported by 2 recent studies showing reduced levels of expression of the mRNA (67) and protein (68) in postmortem brain samples from patients with schizophrenia.

Dysbindin 1 binds both $\alpha$ - and $\beta$-dystrobrevin, which are components of the dystrophin glycoprotein complex. The dystrophin complex is found in the sarcolemma of muscle but is also located in postsynaptic densities in a number of brain areas, particularly mossy fiber synaptic terminals in the cerebellum and hippocampus. Although its functions are largely unknown, its location initially suggested that variation in DTNBP1 might confer risk of schizophrenia by mediating effects on postsynaptic structure and function (58). However, Talbot and colleagues (68) have recently shown that the presynaptic dystrobrevin-independent fraction of dysbindin is reduced in the schizophrenic brain within certain intrinsic glutamatergic neurones of the hippocampus and that this is associated with increased expression of vesicular glutamate transporter type 1 . Moreover, a reduction in glutamate release has been demonstrated in cultured neurons with reduced DTNBP1 expression (64). These data suggest that variation in DTNBP1 might confer risk by altering presynaptic glutamate function.

Neuregulin 1. Neuregulin 1 (NRG1) was implicated in schizophrenia by a study in an Icelandic sample (50). Association analysis across 8p21-22 revealed highly significant evidence for association between schizophrenia and a multimarker haplotype at the $5^{\prime}$ end of NRG1. Strong evidence for association with the same haplotype was subsequently found in a large sample from Scotland (69) and weakly replicated in a United Kingdom sample (70). Further positive findings have emerged from Irish (71), Chinese (72-75), and South African (76) samples. However, some negative findings have also been reported (76-79). Only the 3 studies of Icelandic, Scottish, and UK samples $(50,69,70)$ have implicated the specific Icelandic haplotype, perhaps reflecting differences in the linkage disequilibrium (LD) structure across NRG1 in European and Asian samples (74).

Despite detailed resequencing (50), it has not yet proven possible to identify specific susceptibility variants, but the Icelandic haplotype points to the $5^{\prime}$ end of the gene, once again suggesting that altered expression or perhaps mRNA splicing might be involved. It is even possible at this stage that NRG1 is not itself the susceptibility gene, as intron 1 contains another expressed sequence (71) whose function is unknown. However, insofar as it is possible to model schizophrenia in animals, behavioral analyses of NRG1 hypomorphic mice support the view that the association is related to altered NRG1 function or expression (80). More direct evidence suggesting that altered expression might be involved is also beginning to accumulate (81). Just as for DTNBP1, the mechanisms by which altered NRG1 function might lead to schizophrenia are unclear. NRG1 hypomorphic mice have reduced expression of $\mathrm{N}$-methyl-D-aspartate (NMDA) receptors, suggesting that the effects might be mediated through glutamatergic mechanisms (50). However, NRG1 encodes many mRNA species and proteins; it is thought to encode approximately 15 proteins with a diverse range of functions in the brain, including cell signaling, v-erb-b2 erythroblastic leukemia viral oncogene homologue (ErbB) receptor interactions, axon guidance, synaptogenesis, glial differentiation, myelination, and neurotransmission (82). Any of these could potentially influence susceptibility to schizophrenia.

D-amino acid oxidase and D-amino acid oxidase activator. Chumakov and colleagues (83) undertook association mapping in the linkage region on chromosome $13 q 22-34$. They found associations in French Canadian and Russian populations in markers around 2 novel genes, G72 and G30, which are overlapping but transcribed in opposite directions. G72 is a primate-specific gene expressed in the caudate and amygdala. With the use of yeast 2-hybrid analysis, evidence for physical interaction was found between $G 72$ and $D$-amino acid oxidase (DAO). DAO is expressed in the human brain, where it oxidizes $\mathrm{D}$-serine, a potent activator of NMDA glutamate receptor. Coincubation of G72 and DAO in vitro revealed a functional interaction between the 2, with G72 enhancing the activity of DAO. Consequently, G72 has now been named D-amino acid oxidase activator (DAOA). In the same study, $D A O$ polymorphisms were shown to be associated with schizophrenia in one of the samples, and analysis of DAOA and $D A O$ variants revealed modest evidence for a statistical interaction between the loci and disease risk. Given the 3 levels of interaction, the authors concluded that both genes influence risk of schizophrenia through a similar pathway and that this effect is likely to be mediated through altered NMDA-receptor function.

Associations between DAOA and schizophrenia have subsequently been reported in samples from Germany (84), China (85), and both the US and South Africa (76) as well as in a small sample of very early onset psychosis subjects from the US (86). As before, and conceivably for similar reasons, there is no consensus concerning the specific risk alleles or haplotypes across studies. The German group also reported association between $D A O$ and schizophrenia, although with no evidence for a statistical interaction between the loci and risk of schizophrenia. At present, the published genetic evidence in support of this gene is weaker than that for DTNBP1 and NRG1.

Regulator of $G$ protein signaling 4. Another gene associated with schizophrenia is Regulator of G protein signaling 4 (RGS4), which maps to the putative linkage region on chromosome $1 \mathrm{q} 22$. It was targeted for genetic analysis (87) following a microarray-based geneexpression study in which decreased RGS4 expression was found in schizophrenic postmortem brain. Independent evidence for association between schizophrenia and a haplotype at the $5^{\prime}$ end of the gene was found in 2 samples from the US, and while it did not provide significant evidence alone, a sample from India that was included added to the overall level of support. Positive findings have subsequently been reported by several other groups (88-90), but the level of support for each has been modest and the pattern of association different among samples. RGS4 is a negative regulator of $G$ protein-coupled receptors. However, the evidence that RGS4 modulates activity at certain serotonergic (91) and metabotropic glutamatergic receptors (92) while its own expression is modulated 


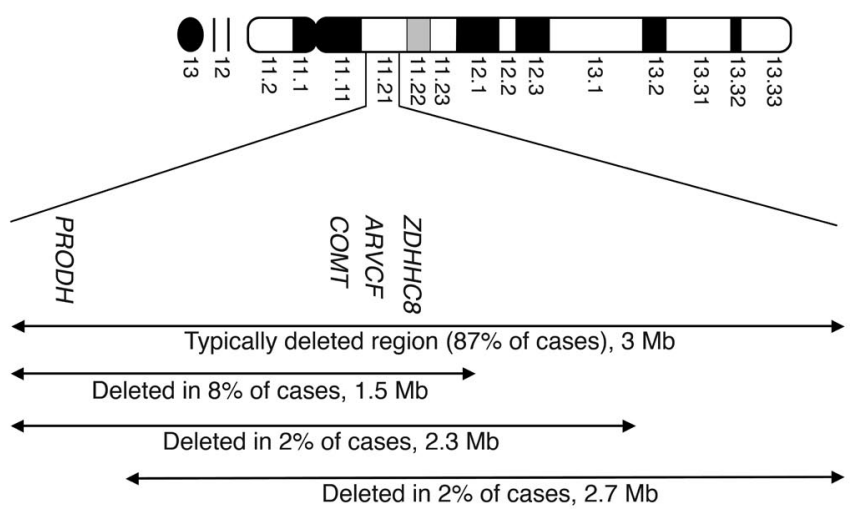

Figure 3

Chromosome 22 and the location of the VCFS deletions. The positions of candidate genes within the typically deleted region that are discussed in this paper are also indicated. The frequencies of the deleted regions are taken from Shaikh et al. (103).

by dopaminergic neurotransmission is of relevance to its possible role in schizophrenia (93). Moreover, RGS4 interacts with ErbB3 (94), which may be of relevance as ErbB3 is a NRG1 receptor whose expression is downregulated in schizophrenic brains (82).

Other positional candidate genes. Association analysis in single studies of CAPON (C terminal PDZ domain ligand of neuronal nitric oxide synthase) (95), PPP3CC (protein phosphatase 3 , catalytic subunit) (96), and trace amine receptor 4 (TRAR4) (97), which map to the $1 \mathrm{q} 22,8 \mathrm{p} 21.3$, and $6 \mathrm{q} 23.2$ linkage regions, respectively, has suggested these as possible susceptibility genes. As discussed in the original articles, each of these genes can be plausibly related to candidate pathophysiologies of schizophrenia, but at this writing, we are not aware of any replication data to support these hypotheses.

\section{Chromosomal abnormalities}

There have been numerous reports of associations between schizophrenia and chromosomal abnormalities (98), but with 2 exceptions, none provides convincing evidence for the location of a susceptibility gene. Several studies have shown that adults with $22 \mathrm{q} 11$ deletions have a high risk of schizophrenia (99-101), with the largest study of adult patients to date $(n=50)$ estimating this at $24 \%(101)$. The deletion cannot account for a high proportion of schizophrenia cases (102), but reports of linkage to 22q11 $(55,56)$ suggest that variants in genes mapping to this region might contribute to cases of schizophrenia that do not have 22q11 deletions. Current candidates include catecholamine O-methyl transferase (COMT), proline dehydrogenase $(P R O D H)$, and zinc finger- and DHHC domain-containing protein 8 ( $Z D H H C 8)$. The positions of the deletions on $22 \mathrm{q} 11$ and their frequencies (103) are shown in Figure 3.

COMT has been intensively studied because of its key role in dopamine catabolism. Most studies have focused upon a valineto-methionine change at codon 158 of the brain-predominant membrane-bound form of COMT (MB-COMT) and codon 108 of the soluble form (S-COMT). The valine allele confers higher activity and thermal stability to COMT (104) and has been fairly consistently associated with reduced performance in tests of frontal lobe function $(105,106)$. The results for schizophrenia have been mixed, with a recent metaanalysis (107) reporting no overall evidence for association with the valine allele.
Since the preparation of the metaanalysis, an Israeli study of over 700 cases reported strong evidence for association between haplotypes, including the val158 allele and 2 flanking, noncoding SNPs (108). As in an earlier study (109), the evidence from haplotypes was stronger than for the valine allele alone, which suggests that COMT may well be a susceptibility gene for schizophrenia, but that the effect is not attributable to the valine/methionine variant. We have been unable to replicate association with any of the SNPs or halotypes, including the valine/methionine polymorphism, in a study of more than 2800 individuals including almost 1200 schizophrenics (Williams, et al. in press; ref. 110), but 2 other groups $(104,111)$ have recently reported rather different haplotype associations at COMT in Irish and US samples. As for the Israeli study, haplotypes carrying the val158 allele exhibited stronger evidence for association than did that allele alone while in the second study, the strongest findings included markers spanning the $3^{\prime}$ end of the armadillo repeat deleted in velocardiofacial syndrome gene $(A R V C F)$. The latter has also been implicated in an earlier study (109), and its transcribed genomic sequence overlaps with that of COMT (112). While the picture is confused, we believe that the evidence does not support a role for valine/methionine 158 in susceptibility to schizophrenia, although a small effect cannot be excluded nor can a role in phenotype modification. However, it remains a strong possibility that variation elsewhere in COMT or in $A R V C F$ confers susceptibility.

$P R O D H$ is another functional candidate gene, given that a lossof-function mutant mouse exhibits behavioral abnormalities in sensorimotor gating that are analogous to those observed in patients with schizophrenia (113) and that PRODH influences the availability of glutamate. In addition, a heterozygous deletion of the entire PRODH gene was found in a family that included 2 subjects with schizophrenia, and 2 heterozygous $P R O D H$ missense variants were detected in 3 of 63 patients with schizophrenia studied by Jacquet et al. (114). Evidence in favor of association between $P R O D H$ and schizophrenia has been reported by Liu and colleagues (115) and by Li and colleagues (116). We have been unable to replicate either of these findings in large case-control and family-based association samples (117). Moreover, we have also observed a range of missense mutations that are equally common in patients with schizophrenia and controls (117); and in a very large sample of Japanese subjects, $P R O D H$ deletions were not associated with schizophrenia (118).

Finally, there is evidence that a SNP in ZDHHC8, a gene which encodes a putative transmembrane palmitoyl transferase, directly confers susceptibility to schizophrenia in females (119). The genetic evidence is not strong but has gained circumstantial support from data suggesting that this SNP may affect the splicing of an mRNA expressed in brain regions relevant to schizophrenia (119). Unfortunately, the only published attempt at replication so far found the opposite allele to be associated and no evidence for a gender effect (120).

The other major finding based upon a chromosomal abnormality comes from an extended pedigree in which a balanced chromosomal translocation $(1,11)(\mathrm{q} 42 ; \mathrm{q} 14.3)$ showed strong evidence for linkage to a fairly broad phenotype consisting of schizophrenia, bipolar disorder, and recurrent depression (36). The translocation was found to disrupt 2 genes on chromosome 1: DISC1 and DISC2 $(36,121)$. DISC2 contains no open reading frame and may regulate DISC1 expression via antisense RNA (121). Interestingly, DISC1 and DISC2 are located close 
to the chromosome 1 markers implicated in 2 Finnish linkage studies $(35,37)$ (Figure 1). It has been suggested that truncation of DISC1 in the translocation family might contribute to schizophrenia by affecting neuronal functions dependent upon intact cytoskeletal regulation, such as neuronal migration, neurite architecture, and intracellular transport $(122,123)$. While these are interesting hypotheses, it is important to remember that translocations can exert effects on genes other than those directly disrupted. For example, there are several mechanisms by which a translocation can influence the expression of neighboring genes. Thus, in order to unequivocally implicate DISC1 and/ or DISC2 in the pathogenesis of schizophrenia, it is necessary to identify in another population mutations or polymorphisms that are associated with schizophrenia that are not in linkage disequilibrium with neighboring genes. Four published studies have attempted to do this; negative findings were reported by the Edinburgh group that originally identified DISC1 and DISC2 (124) and by a group that focused on the $5^{\prime}$ end of the gene in a large Japanese sample (125). However, positive findings have been reported in a large Finnish sample (see supplemental ref. $\mathrm{S} 1$; supplemental references available online with this article; doi:10.1172/JCI24759DS1) and in US samples with schizophrenia, schizoaffective disorder, or bipolar disorder (S2) At present, therefore, the genetic evidence in favor of DISC1 as a susceptibility gene for schizophrenia is gaining weight but, in our view, is not yet compelling. In addition, there are suggestions that DISC1 variants might confer susceptibility to a range of phenotypes, including schizoaffective disorder, bipolar disorder, and recurrent major depressive disorder as well as schizophrenia.

\section{Functional candidate genes}

There is a huge schizophrenia candidate-gene literature consisting of negative findings or positive findings that have either not been replicated or have seemed so unconvincing that there have been no attempts to replicate them. While most of the reported positives are unlikely to stand the test of time, neither can we conclude that any gene has been effectively excluded, given that most have not been studied exhaustively in large samples through a combination of detailed sequencing of exons, introns, and large regions of $5^{\prime}$ and 3 ' flanking sequences combined with exhaustive genotyping. However, metaanalyses do at least suggest that the dopamine receptors DRD3 (S3) and DRD2 (S4) and the serotonergic receptor HTR2A (S5) might confer risk. The effect sizes, if any, are extremely small with odds ratios (ORs) less than 1.2 and difficult to confirm, but if the associations exist, the findings provide some support for the view that altered dopaminergic and serotonergic function might be a primary event in susceptibility to schizophrenia. If the putative associations are the result of linkage disequilibrium between the assayed markers and the true susceptibility variant, it is possible that the latter might contribute somewhat more, depending upon the degree of LD between the 2 .

There is accumulating evidence for abnormalities of glutamatergic neurotransmission in schizophrenia. By and large, analysis of glutamatergic genes as functional candidates for schizophrenia has failed to produce consistent positive findings. However, recently claims have been made that variation in GRM3 (the gene encoding metabotropic glutamate receptor-type 3) might confer risk of schizophrenia. GRM3 was first implicated in a case-control study of German patients in which one SNP was significantly associated with schizophrenia $(P=0.0022)$ in 1 out of the 3 groups of patients studied (S6). Fujii and colleagues (S7) genotyped 6 SNPs in a small case-control sample from Japan. They found significant association with one SNP $(P=0.011$, uncorrected $)$ and a 3-marker haplotype containing this SNP $\left(P=8.30 \times 10^{-4}\right)$. Egan et al. (S8) genotyped 7 SNPs, including the 2 implicated in the previous reports, in a familybased association study. Weak evidence for overtransmission to cases was observed for one SNP $(P=0.02$, uncorrected). However this was not one of the SNPs implicated previously, and the result failed to replicate in a second sample. A haplotype including this SNP was overtransmitted to cases (global $P=0.01$, allele specific $P=0.0001$ ), but again, this did not replicate in a second sample. The authors also presented data relating the single putatively associated GRM3 SNP to cognitive, fMRI, magnetic resonance spectroscopy, and neurochemical variables. They argued that these data all support the conclusion that GRM3 affects prefrontal and hippocampal physiology, cognition, and risk for schizophrenia by altering glutamate neurotransmission. However, the genetic data implicating GRM3 as a susceptibility gene for schizophrenia remain weak, although the findings of Egan and colleagues (S8) clearly suggest that further study is warranted.

Functional implications of susceptibility genes. In our view, the evidence most strongly implicates DTNBP1 and NRG1 as susceptibility genes for schizophrenia, while the data for $D A O, D A O A$, DISC1, and RGS4 are promising but not yet compelling. Even in the most convincing cases, the risk haplotypes appear to be associated with small effect sizes $(\mathrm{OR}<2.5)$ and, although this is difficult to determine, do not appear to fully explain the linkage findings that prompted each study. This could suggest that the associated polymorphisms/haplotypes are only in weak LD with the true pathogenic variants, that the linkages reflect variation at more than one susceptibility site in the same gene (or in multiple genes in the area of linkage), or that in some cases, despite the statistical evidence, the associations are spurious. Detailed followup studies, including de novo mutation detection and detailed genotyping in large samples drawn from different populations, with the aim of answering these questions are now required.

For most geneticists, the purpose of gene identification is the enhancement of our understanding of the pathogenesis of the disorder in question, and in this respect, the genes identified by positional genetics offer the most unbiased insights. Given the strength of the evidence for some genes, it is now important to identify the specific mechanisms by which the implicated genes alter schizophrenia risk and to identify the molecular and cognitive processes that link these primary events to psychopathology. It has been noted that several of the genes encode proteins that potentially have an impact on the function of glutamatergic synapses (S9-S11), leading to the hypothesis that the primary abnormality in schizophrenia is the synapse, particularly the glutamatergic synapse. The possible importance of synaptic abnormalities in schizophrenia has already been recognized (S12), and we now appear to be at a point of convergence between the genetics of schizophrenia and its neurobiology. However, there are strong reasons for remaining cautious: (a) The genetic evidence is not yet definitive, and we have not identified the specific pathogenic variants, much less the pathogenic mechanisms; (b) If we consider the 2 best-supported genes, NRG1 encodes proteins with multiple functions of potential relevance to alternative hypotheses of schizophrenia, for example, aberrant myelination (82), while the function(s) of dysbindin are still obscure; (c) The widely held assumption that schizophrenia is 
a heterogeneous disorder with more that 1 core pathophysiology may well be correct. The desire, then, to fit the data into a unified theory is attractive and parsimonious, in which case it will not be possible to fit the data into a unified theory. It is therefore vital that there be no letup in the hunt for novel schizophrenia genes, the finding of which will allow us to test existing and to generate novel hypotheses of schizophrenia pathogenesis.

\section{Conclusions}

Molecular genetic studies of schizophrenia are built upon the firm foundations of reliable diagnostic methodology and a wealth of genetic epidemiological data. The fact that most, if not all, disease genes apparently have only moderate or small effect sizes has proven challenging, as with other common diseases. However, the resources and reagents generated by the human genome project have begun to make adequately powered studies feasible. A number of potential regions of linkage and 2 associated chromosomal abnormalities have been identified, and accumulating evidence favors several positional candidate genes, although in no case has the causative variant(s) been unequivocally identified. These findings are grounds for optimism and suggest that, while we still have far to travel, we are at least moving in the right direction.

Address correspondence to: Michael Owen, Department of Psychological Medicine, Wales College of Medicine, Cardiff University, Henry Wellcome Building, Heath Park, Cardiff CF14 4XN, United Kingdom. Phone: 44-2920-743248; Fax: 44-2920-746554; E-mail: owenmj@cardiff.ac.uk.
1. McGrath, J., et al. 2004. A systematic review of the incidence of schizophrenia: the distribution of rates and the influence of sex, urbanicity, migrant status and methodology. BMC Med. 2:13.

2. McGuffin, P., Owen, M.J., O’Donovan, M.C., Thapar, A., and Gottesman, I.I. 1994. Schizophrenia. In Seminars in psychiatric genetics, Royal College of Psychiatrists. London, United Kingdom. 87-109.

3. Gottesman, I.I. 1991. Schizophrenia genesis: the origins of madness. Henry Holt \& Company Inc. New York, New York, USA. 296 pp

4. Riley, B.C., and Kendler, K.S. 2004. Schizophrenia genetics. In Kaplan and Sadock's comprehensive text book psychiatry. 8th edition. B.J. Sadock and V.A Sadock, editors. Lippincott Williams and Wilkins. Philadelphia, Pennsylvania, USA. 1354-1371.

5. Cardno, A.G., and Gottesman, I.I. 2000. Twin studies of schizophrenia: from bow-and-arrow concordances to star war Mx and functional genomics. Am. J. Med. Genet. 97:12-17.

6. Kyvik, K.O., Green, A., and Beck-Nielsen, H. 1995 Concordance rates of insulin dependent diabetes mellitus: a population based study of young Danish twins. BMJ. 311:913-917.

7. Hyttinen, V., Kaprio, J., Kinnunen, L., Koskenvuo, M., and Tuomilehto, J. 2003. Genetic liability of type 1 diabetes and the onset age among 22,650 young Finnish twin pairs: a nationwide follow-up study. Diabetes. 52:1052-1055.

8. Locatelli, I., Lichtenstein, P., and Yashin, A.I. 2004. The heritability of breast cancer: a bayesian correlated frailty model applied to Swedish twins data. Twin Res. 7:182-191.

9. Zdravkovic, S., et al. 2002. Heritability of death from coronary heart disease: a 36-year follow-up of 20966 Swedish twins. J. Intern. Med. 252:247-254.

10. Poulsen, P., Kyvik, K.O., Vaag, A., and Beck-Nielsen, H. 1999. Heritability of type II (non-insulin-dependent) diabetes mellitus and abnormal glucose tolerance-a population-based twin study. Diabetologia. 42:139-145

11. Gottesman, I.I., and Shields, J. 1967. A polygenic theory of schizophrenia. Proc. Natl. Acad. Sci.U. S. A. 58:199-205.

12. McGue, M., and Gottesman, I.I. 1989. A single dominant gene still cannot account for the transmission of schizophrenia. Arch. Gen. Psychiatry. 46:478-480.

13. Risch, N. 1990. Genetic linkage and complex diseases, with special reference to psychiatric disorders [review]. Genet. Epidemiol. 7:3-16; discussion $17-45$.

14. Gottesman, I.I., and Shields, J. 1982. Schizophrenia: the epigenetic puzzle. Cambridge University Press. Cambridge, United Kingdom. 258 pp.

15. Farmer, A.E., McGuffin, P., and Gottesman I.I. 1987. Twin concordance for DSM-III schizophrenia. Scrutinizing the validity of the definition. Arch. Gen. Psychiatry. 44:634-641.
16. Kendler, K.S., Neale, M.C., and Walsh, D. 1995. Evaluating the spectrum concept of schizophrenia in the Roscommon Family Study. Am. J. Psychiatry. 152:749-754.

17. Kendler, K.S., Karkowski, L.M., and Walsh, D. 1998. The structure of psychosis: latent class analysis of probands from the Roscommon Family Study. Arch. Gen. Psychiatry. 55:492-499.

18. Tienari, P. et al. 2000. Finnish adoptive family study: sample selection and adoptee DSM-III-R diagnoses. Acta Psychiatr. Scand. 101:433-443.

19. Murray, R.M., Jones, P.B., Susser, E., van Os, J., and Cannon, M. 2002. The epidemiology of schizophrenia. Cambridge University Press. Cambridge, United Kingdom. 454 pp.

20. Seeman, P., and Lee, T. 1975. Antipsychotic drugs: direct correlation between clinical potency and presynaptic action on dopamine neurons. Science. 188:1217-1219.

21. Abi-Dargham, A., et al. 2000. Increased baseline occupancy of D2 receptors by dopamine in schizophrenia. Proc. Natl. Acad. Sci. U. S. A. 97:8104-8109.

22. Weinberger, D.R., et al. 2001. Prefrontal neurons and the genetics of schizophrenia. Biol. Psychiatry. 50:825-844.

23. Meltzer, H.Y., Matsubara, S., and Lee, J.C. 1989. Classification of typical and atypical antipsychotic drugs on the basis of dopamine D-1, D-2 and serotonin 2 pKi values. J. Pharmacol. Exp. Ther. 251:238-246

24. Javitt, D.C., and Zukin, S.R. 1991. Recent advances in the phencyclidine model of schizophrenia. Am. J. Psychiatry. 148:1301-1308.

25. Laruelle, M., Kegeles, L.S., and Abi-Dargham, A 2003. Glutamate, dopamine, and schizophrenia: from pathophysiology to treatment. Ann. N.Y. Acad. Sci. 1003:138-158.

26. Weinberger, D.R. 1995. From neuropathology to neurodevelopment. Lancet. 346:552-557.

27. Murray, R.M., and Lewis, S.W. 1987. Is schizophrenia a neurodevelopmental disorder? Br. Med. J. 295:681-682

28. Pantelis, C., et al. 2003. Neuroanatomical abnormalities before and after onset of psychosis: a cross-sectional and longitudinal MRI comparison. Lancet. 361:281-288.

29. Lawrie, S.M., et al. 1999. Magnetic resonance imaging of brain in people at high risk of developing schizophrenia. Lancet. 353:30-33.

30. Harrison, P.J. 1999. The neuropathology of schizophrenia. A critical review of the data and their interpretation. Brain. 122:593-624.

31. Davis, K.L., et al. 2003. White matter changes in schizophrenia: evidence for myelin-related dysfunction. Arch. Gen. Psychiatry. 60:443-456.

32. Suarez, B.K., Hampe, C.L., and Van Eerdewegh, P. 1994. Problems of replicating linkage claims in psychiatry. In Genetic approaches to mental disorders. E.S. Gershon and C.R. Cloninger, editors. American
Psychiatric Press. Washington, DC, USA. 23-46.

33. Lander, E., and Kruglyak, L. 1995. Genetic dissection of complex traits: guidelines for interpreting and reporting linkage results. Nat. Genet. 11:241-247.

34. Brzustowicz, L.M., Hodgkinson, K.A., Chow, E.W., Honer, W.G., and Bassett, A.S. 2000. Location of a major susceptibility locus for familial schizophrenia on chromosome 1q21-q22. Science. 288:678-682.

35. Ekelund, J., et al. 2001. Chromosome 1 loci in Finnish schizophrenia families. Hum. Mol. Genet. 10:1611-1617.

36. Blackwood, D.H., et al. 2001. Schizophrenia and affective disorders-cosegregation with a translocation at chromosome 1q42 that directly disrupts brain-expressed genes: clinical and P300 findings in a family. Am. J. Hum. Genet. 69:428-433.

37. Ekelund, J., et al. 2004. Replication of 1q42 linkage in Finnish schizophrenia pedigrees. Mol. Psychiatry. 9:1037-1041.

38. Gurling, H.M., et al. 2001. Genomewide genetic linkage analysis confirms the presence of susceptibility loci for schizophrenia, on chromosomes $1 \mathrm{q} 32.2,5 \mathrm{q} 33.2$, and $8 \mathrm{p} 21-22$ and provides support for linkage to schizophrenia, on chromosomes 11q23.3-24 and 20q12.1-11.23. Am. J. Hum. Genet. 68:661-673.

39. Straub, R.E., MacLean, C.J., O’Neil, F.A., Walsh, D., and Kendler, K.S. 1997. Support for a possible schizophrenia vulnerability locus in region 5 q2231 in Irish families. Mol. Psychiatry. 2:148-155.

40. Devlin, B., et al. 2002. Genome-wide multipoint linkage analyses of multiplex schizophrenia pedigrees from the oceanic nation of Palau. Mol. Psychiatry. 7:689-694.

41. Paunio, T., et al. 2001. Genome-wide scan in a nationwide study sample of schizophrenia families in Finland reveals susceptibility loci on chromosomes 2q and 5q. Hum. Mol. Genet. 10:3037-3048.

42. Straub, R.E., et al. 1995. A potential vulnerability locus for schizophrenia on chromosome 6p24-22: evidence for genetic heterogeneity. Nat. Genet. 11:287-293.

43. Schizophrenia Linkage Collaborative Group for Chromosomes 3, 6, and 8. 1996. Additional support for schizophrenia linkage on chromosomes 6 and 8: a multicenter study. Am. J. Med. Genet. 67:580-594.

44. Maziade, M., Bissonnette, L., Rouillard, E., Roy, M.A., and Merette, C. 1997. Further evidence of a susceptibility gene for schizophrenia in 6 p22-p24: a contribution from the eastern Quebec population. Am. J. Med. Genet. 74:666-667.

45. Schwab, S.G., et al. 2000. A genome-wide autosomal screen for schizophrenia susceptibility loci in 71 families with affected siblings: support for loci on chromosome $10 \mathrm{p}$ and 6. Mol. Psychiatry. 5:638-649. 
46. Martinez, M., et al. 1999. Follow-up study on a susceptibility locus for schizophrenia on chromosome 6q. Am. J. Med. Genet. 88:337-343.

47. Lindholm, E. et al. 2001. A schizophrenia-susceptibility locus at $6 \mathrm{q} 25$, in one of the world's largest reported pedigrees. Am. J. Hum. Genet. 69:96-105.

48. Lerer, B., et al. 2003. Genome scan of Arab Israeli families maps a schizophrenia susceptibility gene to chromosome $6 \mathrm{q} 23$ and supports a locus at chromosome 10q24. Mol. Psychiatry. 8:488-498.

49. Blouin, J.L., et al. 1998. Schizophrenia susceptibility loci on chromosomes $13 \mathrm{q} 32$ and $8 \mathrm{p} 21$. Nat. Genet. 20:70-73.

50. Stefansson, H., et al. 2002. Neuregulin 1 and susceptibility to schizophrenia. Am. J. Hum. Genet. 71:877-892.

51. DeLisi, L.E., et al. 2002. A genome-wide scan for linkage to chromosomal regions in 382 sibling pairs with schizophrenia or schizoaffective disorder. Am. J. Psychiatry. 159:803-812.

52. Fallin, M.D., et al. 2003. Genomewide linkage scan for schizophrenia susceptibility loci among Ashkenazi Jewish families shows evidence of linkage on chromosome 10q22. Am. J. Hum. Genet. 73:601-611.

53. Williams, N., et al. 2003. A systematic genomewide linkage study in $353 \mathrm{sib}$ pairs with schizophrenia. Am. J. Hum. Genet. 73:1355-1367.

54. Brzustowicz, L.M., et al. 1999. Linkage of familial schizophrenia to chromosome $13 \mathrm{q} 32$. Am. J. Hum. Genet. 65:1096-1103.

55. Badner, J.A., and Gershon, E.S. 2002. Meta-analysis of whole-genome linkage scans of bipolar disorder and schizophrenia. Mol. Psychiatry. 7:405-411.

56. Lewis, C.M., et al. 2003. Genome scan meta-analysis of schizophrenia and bipolar disorder, part II: Schizophrenia. Am. J. Hum. Genet. 73:34-48.

57. Gottesman, I.I., and Gould, T.D. 2003. The endophenotype concept in psychiatry: etymology and strategic intentions. Am. J. Psychiatry. 160:636-645.

58. Straub, R.E., et al. 2002. Genetic variation in the 6 p22.3 gene DTNBP1, the human ortholog of the mouse dysbindin gene, is associated with schizophrenia. Am. J. Hum. Genet. 71:337-348.

59. Schwab, S.G., et al. 2003. Support for association of schizophrenia with genetic variation in the $6 \mathrm{p} 22.3$ gene, dysbindin, in sib-pair families with linkage and in an additional sample of triad families. Am.J. Hum. Genet. 72:185-190.

60. Williams, N.M., et al. 2004. Identification in 2 independent samples of a novel schizophrenia risk haplotype of the dystrobrevin binding protein gene (DTNBP1). Arch. Gen. Psychiatry. 61:336-344.

61. Kirov, G., et al. 2004. Strong evidence for association between the dystrobrevin binding protein 1 (DTNBP1) gene and schizophrenia in 488 parent-offspring trios from Bulgaria. Biol. Psychiatry. 55:971-975.

62. Van Den Bogaert, A., et al. 2003. The DTNBP1 (dysbindin) gene contributes to schizophrenia, depending on family history of the disease. Am.J. Hum. Genet. 73:1438-1443.

63. Morris, D.W., et al. 2003. No evidence for association of the dysbindin gene [DTNBP1] with schizophrenia in an Irish population-based study. Schizophr. Res. 60:167-172.

64. Numakawa, T., et al. 2004. Evidence of novel neuronal functions of dysbindin, a susceptibility gene for schizophrenia. Hum. Mol. Genet. 13:2699-2708.

65. Funke, B., et al. 2004. Association of the DTNBP1 locus with schizophrenia in a U.S. Population. Am. J. Hum. Genet. 75:891-898.

66. Bray, N.J., Buckland, P.R., Owen, M.J., and O'Donovan, M.C. 2003. Cis-acting variation in the expression of a high proportion of genes in human brain. Hum. Genet. 113:149-153.

67. Weickert, C.S., et al. 2004. Human dysbindin
(DTNBP1) gene expression in normal brain and in schizophrenic prefrontal cortex and midbrain. Arch. Gen. Psychiatry. 61:544-555.

68. Talbot, K., et al. 2004. Dysbindin-1 is reduced in intrinsic, glutamatergic terminals of the hippocampal formation in schizophrenia. J. Clin. Invest. 113:1353-1363. doi:10.1172/JCI200420425.

69. Stefansson, H., et al. 2003. Association of neuregulin 1 with schizophrenia confirmed in a Scottish population. Am. J. Hum. Genet. 72:83-87.

70. Williams, N.M., et al. 2003. Support for genetic variation in neuregulin 1 and susceptibility to schizophrenia. Mol. Psychiatry. 8:485-487.

71. Corvin, A.P., et al. 2004. Confirmation and refinement of an 'at-risk' haplotype for schizophrenia suggests the EST cluster, Hs.97362, as a potential susceptibility gene at the neuregulin-1 locus. Mol. Psychiatry. 9:208-213.

72. Yang, J.Z., et al. 2003. Association study of neuregulin 1 gene with schizophrenia. Mol. Psychiatry. 8:706-709.

73. Tang, J.X., et al. 2004. Polymorphisms within $5^{\prime}$ end of the neuregulin 1 gene are genetically associated with schizophrenia in the Chinese population. Mol. Psychiatry. 9:11-12.

74. Zhao, X., et al. 2004. A case control and family based association study of the neuregulin 1 gene and schizophrenia. J. Med. Genet. 41:31-34.

75. Li, T., et al. 2004. Identification of a novel neuregulin 1 at-risk haplotype in Han schizophrenia Chinese patients, but no association with the Icelandic/Scottish risk haplotype. Mol. Psychiatry. 9:698-704.

76. Hall, D., Gogos, J.A., and Karayiorgou, M. 2004 The contribution of three strong candidate schizophrenia susceptibility genes in demographically distinct populations. Genes Brain Behav. 3:240-248.

77. Iwata, N., et al. 2004. No association with the neuregulin 1 haplotype to Japanese schizophrenia. Mol. Psychiatry. 9:126-127.

78. Thiselton, D.L., et al. 2004. No evidence for linkage or association of neuregulin-1 (NRG1) with disease in the Irish study of high-density schizophrenia families (ISHDSF). Mol. Psychiatry. 9:777-783.

79. Hong, C.J., et al. 2004. Case-control and familybased association studies between the neuregulin 1 (Arg38Gln) polymorphism and schizophrenia. Neurosci. Lett. 366:158-161.

80. Stefansson, H., Steinthorsdottir, V., Thorgeirsson, T.E., Gulcher, J.R., and Stefansson, K. 2004. Neuregulin 1 and schizophrenia. Ann. Med.36:62-71.

81. Hashimoto, R., et al. 2004. Expression analysis of neuregulin-1 in the dorsolateral prefrontal cortex in schizophrenia. Mol. Psychiatry. 9:299-307.

82. Corfas, G., Roy, K., and Buxbaum, J.D. 2004. Neuregulin 1-erbB signaling and the molecular/cellular basis of schizophrenia. Nat. Neurosci. 7:575-580.

83. Chumakov, I., et al. 2002. Genetic and physiological data implicating the new human gene G72 and the gene for D-amino acid oxidase in schizophrenia. Proc. Natl. Acad. Sci. U. S. A. 99:13675-13680.

84. Schumacher, J., et al. 2004. Examination of G72 and D-amino-acid oxidase as genetic risk factors for schizophrenia and bipolar affective disorder. Mol. Psychiatry. 9:203-207.

85. Wang, X., et al. 2004. Association of G72/G30 with schizophrenia in the Chinese population. Biochem. Biophys. Res. Commun. 319:1281-1286.

86. Addington, A.M., et al. 2004. Polymorphisms in the 13 q33.2 gene G72/G30 are associated with childhood-onset schizophrenia and psychosis not otherwise specified. Biol. Psychiatry. 55:976-980.

87. Chowdari, K.V., et al. 2002. Association and linkage analyses of RGS4 polymorphisms in schizophrenia. Hum. Mol. Genet. 11:1373-1380.

88. Williams, N.M., et al. 2004. Support for RGS4 as a susceptibility gene for schizophrenia. Biol. Psychiatry. 55:192-195.
89. Chen, X., et al. 2004. Regulator of G-protein signaling 4 (RGS4) gene is associated with schizophrenia in Irish high density families. Am. J. Med. Genet. B Neuropsychiatr. Genet. 129B:23-26.

90. Morris, D.W., et al. 2004. Confirming RGS4 as a susceptibility gene for schizophrenia. Am. J. Med. Genet. B Neuropsychiatr. Genet. 125B:50-53.

91. Beyer, C.E., et al. 2004. Regulators of G-protein signaling 4: modulation of 5-HT(1A)-mediated neurotransmitter release in vivo. Brain Res. 1022:214-220.

92. De Blasi, A., Conn, P.J., Pin, J., and Nicoletti, F. 2001. Molecular determinants of metabotropic glutamate receptor signaling. Trends Pharmacol. Sci. 22:114-120.

93. Taymans, J.M., et al. 2004. Dopamine receptormediated regulation of RGS2 and RGS4 mRNA differentially depends on ascending dopamine projections and time. Eur. J. Neurosci. 19:2249-2260.

94. Thaminy, S., Auerbach, D., Arnoldo, A., and Stagljar, I. 2003. Identification of novel ErbB3interacting factors using the split-ubiquitin membrane yeast two-hybrid system. Genome Res. 13:1744-1753.

95. Brzustowicz, L.M., et al. 2004. Linkage disequilibrium mapping of schizophrenia susceptibility to the CAPON region of chromosome 1q22. Am. J. Hum. Genet. 74:1057-1063.

96. Gerber, D.J., et al. 2003. Evidence for association of schizophrenia with genetic variation in the 8p21.3 gene, PPP3CC, encoding the calcineurin gamma subunit. Proc. Natl. Acad. Sci. U. S. A. 100:8993-8998.

97. Duan, J., et al. 2004. Polymorphisms in the trace amine receptor 4 (TRAR4) gene on chromosome $6 \mathrm{q} 23.2$ are associated with susceptibility to schizophrenia. Am. J. Hum. Genet. 75:624-638.

98. MacIntyre, D.J., Blackwood, D.H., Porteous, D.J., Pickard, B.S., and Muir, W.J. 2003. Chromosomal abnormalities and mental illness. Mol. Psychiatry. 8:275-287.

99. Pulver, A.E., et al. 1994. Psychotic illness in patients diagnosed with velo-cardio-facial syndrome and their relatives. J. Nerv. Ment. Dis. 182:476-478.

100. Bassett, A.S., et al. 1998. 22q11 deletion syndrome in adults with schizophrenia. Am. J. Med. Genet. 81:328-337.

101.Murphy, K.C., Jones, L.A., and Owen, M.J. 1999. High rates of schizophrenia in adults with velocardio-facial syndrome. Arch. Gen. Psychiatry. 56:940-945.

102.Ivanov, D., et al. 2003. A molecular genetic study of the vcfs region in patients with early onset psychosis. Br. J. Psychiatry. 183:409-413.

103.Shaikh, T.H., et al. 2000. Chromosome 22-specific low copy repeats and the 22q11.2 deletion syndrome: genomic organisation and deletion endpoint analysis. Hum. Mol. Genet. 9:489-501.

104.Chen, X., Wang, X., O’Neill, A.F., Walsh, D., and Kendler, K.S. 2004. Variants in the catechol-omethyltransferase (COMT) gene are associated with schizophrenia in Irish high-density families. Mol. Psychiatry. 9:962-967.

105.Egan, M.F., et al. 2001. Effect of COMT Val108/158 Met genotype on frontal lobe function and risk for schizophrenia. Proc. Natl. Acad. Sci. U. S. A. 98:6917-6922.

106. Malhotra, A.K., et al. 2002. A functional polymorphism in the COMT gene and performance on a test of prefrontal cognition. Am. J. Psychiatry. 159:652-654.

107. Glatt, S.J., Faraone, S.V., and Tsuang, M.T. 2003. Association between a functional catechol O-methyltransferase gene polymorphism and schizophrenia: meta-analysis of case-control and family-based studies. Am. J. Psychiatry. 160:469-476.

108. Shifman, S., et al. 2002. A highly significant association between a comt haplotype and schizophrenia. 
Am. J. Hum. Genet. 71:1296-1302.

109.Li, T., et al. 2000. Family-based linkage disequilibrium mapping using SNP marker haplotypes: application to a potential locus for schizophrenia at chromosome 22q11. Mol. Psychiatry. 5:77-84.

110.Williams, H., et al. 2005. No association between polymorphisms in COMT and schizophrenia in two large samples. Am. J. Psychiatry. In press.

111.Sanders, A.R., et al. 2005. Haplotypic association spanning the 22q11.21 genes COMT and ARVCF with schizophrenia. Mol. Psychiatry. 10:353-365.

112.Bray, N.J., et al. 2003. A haplotype implicated in schizophrenia susceptibility is associated with reduced COMT expression in human brain. Am.J. Hum. Genet. 73:152-161.

113.Gogos, J.A., et al. 1999. The gene encoding proline dehydrogenase modulates sensorimotor gating in mice. Nat. Genet. 21:434-439.

114.Jacquet, H., et al. 2002. PRODH mutations and hyperprolinemia in a subset of schizophrenic patients. Hum. Mol. Genet. 11:2243-2249.
115.Liu, H., et al. 2002. Genetic variation at the $22 \mathrm{q} 11$ PRODH/DGCR6 locus presents an unusual pattern and increases susceptibility to schizophrenia. Proc. Natl. Acad. Sci. U. S. A. 99:3717-3722.

116.Li, T., et al. 2004. Evidence for association between novel polymorphisms in the PRODH gene and schizophrenia in a Chinese population. Am. J. Med. Genet. B Neuropsychiatr. Genet. 129B:13-15.

117. Williams, H.J., et al. 2003. Association between PRODH and schizophrenia is not confirmed. Mol. Psychiatry. 8:644-645.

118. Ohtsuki, T., et al. 2004. Failure to find association between PRODH deletion and schizophrenia. Schizophr. Res. 67:111-113.

119.Mukai,J., et al. 2004. Evidence that the gene encoding ZDHHC8 contributes to the risk of schizophrenia. Nat. Genet. 36:725-731.

120.Chen, W.Y., et al. 2004. Case-control study and transmission disequilibrium test provide consistent evidence for association between schizophrenia and genetic variation in the $22 \mathrm{q} 11$ gene
ZDHHC8. Hum. Mol. Genet. 13:2991-2995.

121.Millar, J.K., et al. 2000. Disruption of two novel genes by a translocation co-segregating with schizophrenia. Hum. Mol. Genet. 9:1415-1423.

122. Miyoshi, K., et al. 2003. Disrupted-In-Schizophrenia 1 , a candidate gene for schizophrenia, participates in neurite outgrowth. Mol. Psychiatry. 8:685-694.

123. Ozeki, Y, et al. 2003. Disrupted-in-schizophrenia-1 (DISC-1): mutant truncation prevents binding to NudE-like (NUDEL) and inhibits neurite outgrowth. Proc. Natl. Acad. Sci. U. S. A. 100:289-294.

124.Devon, R.S., et al. 2001. Identification of polymorphisms within Disrupted in Schizophrenia 1 and Disrupted in Schizophrenia 2, and an investigation of their association with schizophrenia and bipolar disorder. Psychiatr. Genet. 11:71-78.

125.Kockelkorn, T.T., et al. 2004. Association study of polymorphisms in the 5 ' upstream region of human DISC1 gene with schizophrenia. Neurosci. Lett. 368:41-45. 